\title{
Article
}

\section{Quantum Field Theory Representation in Quantum Computation}

\author{
Nicolás Lori *D, José Neves and José Machado (D) \\ Centre Algoritmi, University of Minho, 4710-057 Braga, Portugal; jneves@di.uminho.pt (J.N.); \\ jmac@di.uminho.pt (J.M.) \\ * Correspondence: nicolas.lori@algoritmi.uminho.pt; Tel.: +351-253-510-180
}

\section{Featured Application: Quantum Computation.}

\begin{abstract}
Recently, from the deduction of the result $\mathrm{MIP}^{*}=\mathrm{RE}$ in quantum computation, it was obtained that Quantum Field Theory (QFT) allows for different forms of computation in quantum computers that Quantum Mechanics (QM) does not allow. Thus, there must exist forms of computation in the QFT representation of the Universe that the QM representation does not allow. We explain in a simple manner how the QFT representation allows for different forms of computation by describing the differences between QFT and QM, and obtain why the future of quantum computation will require the use of QFT.
\end{abstract}

Keywords: quantum computation; quantum mechanics; quantum field theory

check for

updates

Citation: Lori, N.; Neves, J.;

Machado, J. Quantum Field Theory

Representation in Quantum

Computation. Appl. Sci. 2021, 11,

11272. https://doi.org/10.3390/

app112311272

Academic Editor: Rosario Lo Franco

Received: 15 September 2021

Accepted: 24 November 2021

Published: 28 November 2021

Publisher's Note: MDPI stays neutral with regard to jurisdictional claims in published maps and institutional affiliations.

Copyright: (c) 2021 by the authors. Licensee MDPI, Basel, Switzerland. This article is an open access article distributed under the terms and conditions of the Creative Commons Attribution (CC BY) license (https:// creativecommons.org/licenses/by/ $4.0 /)$.

\section{Introduction}

There have been indications that a Quantum Computer (QC) will not avoid the difficulties existing in a present-day Classical Computer (CC) to preserve the validity of Moore's law [1]; however, recent work has indicated that the quantum computations represented by Quantum Field Theory (QFT) can make computations that are not representable using Quantum Mechanics (QM) [2]. Until the publication of ref. [2], it was usually assumed that Tsirelson's problem had a positive resolution, hence allowing for a computationallyequivalent usage of QFT and QM; however, ref. [2] has shown such a positive resolution is incompatible with the undecidability of Turing's halting problem.

A major difference between QM and QFT is that, for the former, the combined system of two measurement devices is based in the tensor product of the Hilbert space of each of the measurement devices, whereas for the latter, it is assumed that there is a unique Hilbert space for the two measurement devices [2-4]. This difference in the system representation between QM and QFT implies that the method of defining the non-communication of the measurement devices will be different for QM versus for QFT [5].

In QM, this non-communication is represented by each of the possible measurement devices to represent the system being measured by its own Hilbert space, whereas the combined measurement that uses multiple measurement devices is simply the tensor product of the Hilbert space of each of the measurement operators; whereas in QFT, non-communication is expressed by the commutativity of the operators of each of the measurement devices [2,3]. It is therefore possible to differentiate, for a nonlocal game $G$, between the supremum of success probabilities when using a tensor product approach, represented as $\omega^{T P}(G)$; and the supremum of success probabilities when using a commuting operator approach, represented as $\omega^{C O}(G)$.

Until recently, it was assumed in Physics, Mathematics and Computer science that $\omega^{T P}(G)=\omega^{C O}(G)$, which was the positive resolution of Tsirelson's problem and is equivalent to the positive resolution of Connes' embedding problem $[2,6]$. However, recent work has demonstrated that $\omega^{T P}(G)<\omega^{C O}(G)$ [2], which implied that the QC-limiting results obtained (e.g., ref. [1]) could perhaps be overcome by results obtained using QFT 
representations, and this is what we will analyze in this work. Since QFT is a more fundamentally valid representation of the Universe than QM, this implies that the results obtained using a QFT representation are always more realistic than those obtained from a QM representation [4].

A recent result in QFT representations of QCs [2] indicates that although Turing's halting problem is unsolvable, it is verifiable using QFT. This differentiation between verification-computability and resolution-computability is known in CCs; however, in CCs it is a matter of efficiency, whereas in the QFT representation of QC [2] there is a chasm between the two, caused by the occurrence of quantum entanglement. This chasm was already suggested for the QM representation of QC in previous works (e.g., ref. [1]), but in ref. [2], the chasm is demonstrated for the QFT representation of QC.

\section{Materials and Methods}

The comparison between Classical Mechanics (CM) and QM approaches to the Hammersley-Clifford Theorem (HCT) allowed for the comparison between CCs and QCs expressed in ref. [1], which indicated that QCs are likely to out-perform CCs for the physical systems with a small number of physically-allowed alternative future states, but not when that number of possible future states is large. This result of ref. [1] agrees with the result in ref. [2], in that the presence of quantum entanglement allows for a chasm of computability between verifying and finding solutions, as the quantum entanglement allows for the verification of solutions that are unfindable [2,3]. The occurrence of quantum entanglement is thus very useful in the case where there are few alternative future states, but not nearly as useful when there are many alternative future states; hence, this makes QC more useful than $\mathrm{CC}$ for the former but not for the latter, in agreement with the ref. [1] results.

In ref. [1], it is described how for QC the quantum HCT is only capable of having a one-to-one map of the surrounding environment if the sub-Hamiltonians of each of the cliques of the graph representing the environment commute with each other, which implies that the quantum system is in a pure state, which allows that quantum system to make a one-to-one map of its surrounding environment provided that the environment is also in a pure state. However, the $\omega^{T P}(G)<\omega^{C O}(G)$ result of ref. [2] implies that there are nonlocal games, labeled as $G$, and that there are winning strategies for that game in the QFT representation which cannot be represented in the QM representation. Moreover, in the QFT representation, the winning strategy means that the operators commute, whereas in the QM representation it means that the Hilbert space of the quantum system is the tensor product of the Hilbert spaces corresponding to each sub-Hamiltonian. Whereas for the QFT representation of QC, the condition proposed in ref. [1] for the equivalence between environment-representation and environment is performed without the use of the subHamiltonians, and hence the tensor-approach condition must be replaced by the condition that the operators for the different cliques of the quantum system graph commute with each other. Nevertheless, as will be described below, pure states in QFT are not attainable and can only be approximated as idealized simplifications [4].

If the Universe was representable by QM, the difficulties that QCs represented using QFT have in representing external environments will be at least as large as the difficulties that QCs represented using QM have, but the Universe is better represented by QFT than QM. Hence, both the Universe and the QC in the QFT representation are at best in an "almost" pure state. Thus, one might at first consider that by using the QFT representation of the "almost" pure state of the Universe can be represented by an "almost" pure state of the QC; but the difference between the "almost" pure state of QFT and the pure state of $\mathrm{QM}$ is the pattern of creation and destruction of particles in the vacuum state $|0\rangle$, which implies that the random difference between the "almost" pure state and the pure state will be different for the QC and for the Universe. Hence, the difficulties of using a QC to map the surrounding environment by using a QFT representation are larger than the difficulties that arise when using a QM representation. The reason why QFT needs to be used instead 
of $\mathrm{QM}$ is that, at a small enough scale, $\mathrm{QM}$ is no longer a realistic enough approximation and thus QFT needs to be used instead.

The QFT is an extension of QM from low-velocity classical relativity to Einstein's special relativity, but if $m_{0}$ is the rest mass, $\vec{u}=\left(u_{z}, u_{y}, u_{z}\right)$ is the velocity, $c$ is the speed of light in vacuum, $\vec{p}=\left(p_{x}, p_{y}, p_{z}\right)$ is the momentum and $E$ is the energy, the relation between momentum and the energy is very different between classical relativity where for a free particle $E=\frac{|\vec{p}|^{2}}{2 m_{0}}$ and for special relativity where $E^{2}=c^{2}|\vec{p}|^{2}+m_{0}{ }^{2} c^{4}$. This is important because the sobreposition principle of $\mathrm{QM}$ assumes that the relation between energy and momentum must be linear for the energy, which is valid for classic relativity but not special relativity. Dirac resolved that problem and obtained a linear relation between energy and momentum for special relativity, but the consequence was that the energy and each of the three components of the momentum were now a four element spinor, respectively $\bar{E}=\left\{E^{(1)}, E^{(2)}, E^{(3)}, E^{(4)}\right\}, \overrightarrow{\vec{p}}=\left\{\vec{p}^{(1)}, \vec{p}^{(2)}, \vec{p}^{(3)}, \vec{p}^{(4)}\right\}$; so too were the electric potential spinor $\bar{\phi}=\left\{\phi^{(1)}, \phi^{(2)}, \phi^{(3)}, \phi^{(4)}\right\}$ and the vector potential spinor $\overrightarrow{\vec{A}}=\left\{\vec{A}^{(1)}, \vec{A}^{(2)}, \vec{A}^{(3)}, \vec{A}^{(4)}\right\}$ where the electron charge is $-e$. In the notation developed by Dirac usually used in QM, the energy operator is $\mathrm{i} \hbar \frac{d}{d t}$, where $t$ is time and $\hbar$ is the reduced Planck constant, and acts on a four-component vector column $\left\{\psi^{(1)}, \psi^{(2)}, \psi^{(3)}, \psi^{(4)}\right\}$, where each component is a complex function in spacetime. The $\bar{E}$ spinor notation used here simply represents, in each of the four components, the complex function obtained by the action of the energy operator; hence $\bar{E}=\left\{E^{(1)}, E^{(2)}, E^{(3)}, E^{(4)}\right\} \equiv\left\{\mathrm{i} \hbar \frac{d \psi^{(1)}}{d t}, \mathrm{i} \hbar \frac{d \psi^{(2)}}{d t}, \mathrm{i} \hbar \frac{d \psi^{(3)}}{d t}, \mathrm{i} \hbar \frac{d \psi^{(4)}}{d t}\right\}$, and so forth for the other QM operators.

For ease of representation, the $4 \mathrm{D}$ spinors described above, e.g., $\bar{E}$, are divided into two 2D spinors, e.g., for the energy spinor $\bar{E}=\left\{E^{+}, E^{-}\right\}$; with the first indicating the particles and the second indicating the opposite of the particles with the meaning of opposite defined below. Thus, $E^{+} 2 \mathrm{D}$ spinor indicate particles and $E^{-}$indicate the opposite. The physics equations for the QFT are, in this case, the Dirac equation for electromagnetic interactions, which is presented below using our above notation and where the $2 \times 2$ Pauli matrices are represented by $\sigma_{x}, \sigma_{y}$ and $\sigma_{z}$ :

$$
\left\{\begin{array}{c}
\frac{E^{+}}{c} \\
-\frac{E^{-}}{c}
\end{array}\right\}=\left\{\begin{array}{l}
m_{0} c-\frac{e}{c} \phi^{+}+\sigma_{x} \cdot\left(p_{x}^{+}+e A_{x}^{+}\right)+\sigma_{y} \cdot\left(p_{y}^{+}+e A_{y}^{+}\right)+\sigma_{y} \cdot\left(p_{z}^{+}+e A_{z}^{+}\right) \\
m_{0} c+\frac{e}{c} \phi^{-}+\sigma_{x} \cdot\left(p_{x}^{-}+e A_{x}^{-}\right)+\sigma_{y} \cdot\left(p_{y}^{-}+e A_{y}^{-}\right)+\sigma_{z} \cdot\left(p_{z}^{-}+e A_{z}^{-}\right)
\end{array}\right\}
$$

The minus sign in $E^{-}$signifies that it behaves differently from $E^{+}$, but its meaning depends on using either Dirac's or Feynman's perspective. For Dirac, opposite means the absence of a particle, whereas for Feynman, opposite means an anti-particle. The anti-particle is equivalent to a particle moving backward in time with opposite charge $e^{-}=-e^{+}=e$ and opposite spin orientation. The Feynman perspective thus obtains from Equation (1) that:

$\left\{\begin{array}{c}\frac{E^{+}}{c} \\ \frac{E^{-}}{c}\end{array}\right\}=\left\{\begin{array}{l}m_{0} c+\frac{e^{+}}{c} \phi^{+}+\sigma_{x} \cdot\left(p_{x}^{+}-e^{+} A_{x}^{+}\right)+\sigma_{y} \cdot\left(p_{y}^{+}-e^{+} A_{y}^{+}\right)+\sigma_{z} \cdot\left(p_{z}^{+}-e^{+} A_{z}^{+}\right) \\ m_{0} c+\frac{e^{-}}{c} \phi^{-}+\sigma_{x} \cdot\left(p_{x}^{-}-e^{-} A_{x}^{-}\right)+\sigma_{y} \cdot\left(p_{y}^{-}-e^{-} A_{y}^{-}\right)+\sigma_{z} \cdot\left(p_{z}^{-}-e^{-} A_{z}^{-}\right)\end{array}\right\}$

Hence, a direct consequence of making QM compatible with special relativity is for the Feynman perspective: i. the existence of antiparticles, ii. the existence of a particle property called spin, which has the units of action and behaves similarly to a magnetic moment, iii. the linear relation between energy and momentum expressed in Equation (2) has the same form for both particles and anti-particles.

The representation used in QM is usually the Schrödinger picture, where the state of the electron is represented by a complex function called wave-function that covers 
space and evolves through time according to Schrödinger's equation. However, the representation most often used in QFT is an improvement of a different form of representing $\mathrm{QM}$, the Feynman path integral approach. In the Schrödinger picture of QM, the wavefunction square amplitude is a probability density and it has a phase that is dependent on the evolution from the past to the present time, where the evolution is generated by the Hamiltonian operator of the system acting on the wave-function of the system; whereas in the Feynman path representation of QM the same wave-function is created through the addition of all possible paths between the past and the present, with each path occurring in a location $x$ at a time $t$ being generated by a Lagrangian $L_{(x, t)}$ where each Feynman-path step of a mass $m$, associated to a time step of $\Delta t$, has a complex number associated to it approximately equal to $\sqrt{\frac{m}{i 2 \pi \hbar \Delta t}} e^{i \frac{\Delta t}{\hbar} L_{\left(x, t+\frac{\Delta t}{2}\right)}}$. The Heisenberg uncertainty of the Schrödinger picture is represented in the Feynman picture by the integration across all the possible paths to calculate the amplitude and phase of a transition between an initial and final state.

In QFT, the $L_{\left(x, t+\frac{\Delta t}{2}\right)}$ of QM is replaced by an integration of an interaction Lagrangian density $\mathcal{L}_{\text {int }}$ across spacetime. Moreover, the $\sqrt{\frac{m}{22 \pi \hbar \Delta t}} e^{i \frac{\Delta t}{\hbar} L_{\left(x, t+\frac{\Delta t}{2}\right)}}$ step of the Feynman path approach to QM is replaced by the expression in Equation (3). The interaction Lagrangian density $\mathcal{L}_{\text {int }}$ will depend on the quantum fields, and it is the quantum fields that will vary across spacetime. Additionally, in QFT the sum is not performed over paths but over all the time-ordered configurations of the quantum fields, which in short is referred to as the "all configurations" sum. QFT assumes that spacetime coordinates $(\vec{r}, t)$ are flat [4], meaning that the spacetime curvature caused by gravity is very small, and so the phase contribution by spacetime location $\left(\vec{r}^{\prime}, t^{\prime}\right)$ to the time-ordered transition between two quantum field configurations, $\bar{\psi}_{(\vec{r}, t)}$ and $\psi_{(\vec{r}, t+\Delta t)}$, is for QFT (see Appendix A) approximately:

$$
\frac{\left\langle 0\left|\sum_{\text {all configurations }}\left\{\psi_{(\vec{r}, t+\Delta t)} \bar{\psi}_{(\vec{r}, t)} e^{i \frac{\Delta t}{\hbar}[\mathrm{c} \cdot \Delta t]^{3} \mathcal{L}_{\text {int }}\left(\vec{r}^{\prime}, t^{\prime}\right)}\right\}\right| 0\right\rangle}{\left\langle 0\left|\sum_{\text {all configurations }}\left\{e^{i \frac{\Delta t}{\hbar}[\mathrm{c} \cdot \Delta t]^{3} \mathcal{L}_{\text {int }}\left(\vec{r}^{\prime}, t^{\prime}\right)}\right\}\right| 0\right\rangle}
$$

A consequence of Equations (2) and (3) making quantum fields compatible with special relativity is that the particles of the electromagnetic field, the photons, are quanta of the $[\bar{\phi},-c \vec{A}]$ quantum field, and the electrons are quanta of the electron quantum field; therefore, both photons and electrons are being created and destroyed, as much as energy-momentum conservation and Heisenberg's uncertainty rules allow. The breaking of energy-momentum conservation being typically through the occurrence of extremely short-lived virtual particles and virtual antiparticles, which constitute the vacuum state $|0\rangle$ [4]. The existence of a quantum electrodynamics dynamic vacuum state $|0\rangle_{E M}$ has been experimentally confirmed multiple times and is the theoretical basis for cavity quantum electrodynamics which is one of the major approaches towards QC [7].

The establishment of Equations (2) and (3) is the end of QM and the start of QFT, as several of the tenets of QM need to be abandoned. In QM, the state of a particle is represented in a Hilbert space, and the number of required Hilbert spaces grows with the number of particles, and hence there are as many Hilbert spaces as there are particles. However, in QFT the number of particles is dynamic and often undeterminable, and hence the use of Hilbert spaces becomes completely impractical, except for simplistic models where the number of particles is preserved or is irrelevant. Thus, in QFT the quantum fields are considered to exist everywhere, and it is their interaction that can be used to calculate the probability of certain events. Hence, in QFT, the tensor-product approach cannot be used to assess the occurrence of entanglement of quantum systems and instead the commuting-operator approach is used. 
In QM, it is often said that a certain electron is mostly located in a certain region and another electron is located in some other region; however, in QFT those two electrons are indistinguishable quanta manifestations of the same electron quantum field with the two quanta associated to different spacetime locations. In QM, there is only one electron quantum field in the Universe and all electrons are quanta of that field. The electron quantum field is a fermionic quantum field because the electron spin is a semi-integer multiple of $\hbar$, specifically $\frac{1}{2} \cdot \hbar$, which is referred to as being spin $\frac{1}{2}$.

The Standard Model of Physics (SMP) uses the Feynman perspective of QFT to explain all Physics, except for gravity, and when QFT is assessed, it is usually assumed that it is the SMP that is being considered. The SMP consists of bosonic and fermionic quantum fields. The twelve fermionic quantum fields are arranged as three generations, with each generation having four quantum fields, specifically: $\frac{2}{3}$ e charge quark, $-\frac{1}{3} \mathrm{e}$ charge quark, $-e$ charge lepton, and 0 charge neutrino. The electron is the lepton of the first generation, whereas the proton and the neutron are constituted by the first-generation quarks. The bosonic quantum fields are a global gauge-invariant Higgs field, together with local gaugeinvariant quantum fields that are specifically associated to the SU(3), SU(2) and U(1) gauge symmetries. Thus, there are twelve local, gauge bosonic quantum fields each of them with spin $1 \cdot \hbar$, referred to as spin 1 ; the bosonic quantum fields being $3 * 3-1=8$ for $S U(3)$, $2 * 2-1=3$ for $\mathrm{SU}(2)$ and 1 for $\mathrm{U}(1)$; plus the spin 0 Higgs, global, gauge bosonic QF. In short, the SMP of the Universe is constituted by twenty-five quantum fields and all particles are quanta of those quantum fields. Those quantum fields are the twelve quantum fields with spin $\frac{1}{2}$, twelve with spin 1 and one with spin 0 . The vacuum state of the SMP is $|0\rangle_{S M P}$ and has also been experimentally confirmed [4]; moreover, for small enough energies (i.e., large enough wavelengths) it can be affirmed that $|0\rangle_{S M P} \approx|0\rangle_{E M}$.

In the QM representation, a system can be in either a pure state or a mixed state: a pure state is represented by a wave-function whereas a mixed state is represented by a statistical ensemble of pure states. Moreover, two quantum systems that have interacted in the past can stay entangled even as the systems move across spacetime, meaning that the measurement of one of the systems immediately implies the measurement of the system entangled to it. The measurement of a quantum system in a pure state occurs by allowing the entangling of that system to the environment around it: as the entanglement grows in scale, the state of the system becomes first an arbitrary basis mixed state and then a mixed state where each state of the statistical ensemble is an eigenstate of the operator associated to that measurement, meaning that the measurement using an energy operator creates a mixed state of energy eigenstates. In QFT, the vacuum around the system is always causing the spontaneous emission of both particles and virtual particles into and away from the system, hence always creating an entanglement between the system and the environment. Thus, in QFT the systems are always somewhat mixed and always somewhat entangled with their environment. The pure states of QM are hence an oversimplification of what is actually going on in the Universe as described by QFT.

The inexistence of pure states in QFT, except as an idealistic approximation, implies that the use of Hilbert spaces in QFT has an added difficulty to the other already described difficulties. Thus, the tensor product approach of QM cannot be at all applied to QFT, and instead it is necessary to use the commuting operator approach. Notice that in the tensor product approach, the operator's measuring systems that are not entangled commute with each other, implying that in this case there is also nothing in QM representation that is not best described using the QFT representation. The reason for the $\omega^{T P}(G)<\omega^{C O}(G)$ result is now becoming clearer, in QFT there is a spontaneous creation of entanglement that makes the systems become entangled with the $|0\rangle_{S M P}$ vacuum state itself. Hence, by increasing the $\omega^{C O}(G)$ supremum of success probabilities by using commuting operator strategies from QFT above the $\omega^{T P}(G)$ supremum of success probabilities by using tensor product strategies from QM, the QFT approach simply has a greater number of strategies that are not available in the QM approach. 


\section{Results}

We will now assess if these new strategies of the QFT over the QM approach allow for the reduction or increase of computational capacity. The key question in this work is whether the computations allowed by QFT that do not occur in QM can improve the computation capacity of QFs:

(1) QFT perspective affects the capacity of pure states to exist in both the QC and the Universe, which reduces rather than increases the stability of spin states.

(2) The new computation that QFT allows occurs through the interaction between systems and the vacuum state $|0\rangle_{S M P}$, but such an interaction could only improve quantum computation if information could be stored in the vacuum state. This implies that the vacuum states could somehow be programmed. To the best knowledge of contemporary Physics, the vacuum state cannot be programmed.

\section{Discussion}

Due to the new forms of quantum computation allowed by the QFT representation of QCs [2], which we propose to be associated to the dynamic interactions of the quantum fields with the vacuum state, it might appear that a form of improving the computation capacity by use of QFT is to move away from the fermionic electronics described in ref. [1] and to instead focus in bosonic wave confinement technologies, such as those used in integrated optical memories [8]. Although there are different forms for integrated optical memories, e.g., [8] the Master-Slave, Feedback Loop, Injection Locking and Phase-Change Material approaches, they all require the creation of a "box" where light is to be constrained, and that "box" needs to be built by atoms. Thus, it is not surprising that despite recent improvements, they cannot go below the 27,000 nanometer ${ }^{2} 2$ D boxes, which even if we assume a depth of 1 nanometer, would amount to a volume of $30^{3}$ nanometers.

If the volume is a 30-nanometer diameter sphere and the spin orientation of each boson constitutes 1 bit of information, there can be $N$ bits stored in that spherical volume provided the boson quantum field has $N$ polarized quanta each with a different wavelength, with the condition that each wavelength must be equal to $\frac{60}{n}$ nanometer where $n$ is an integer. For the bits stored in the spherical volume to be larger than the information-processing limit described in ref. [1], i.e., 5 bit $/$ nanometer $^{3}$, then $N>5 \times 30^{3}=1.35 \times 10^{5}$ and hence the wavelength would need to be smaller than 0.44 picometer which is a scale between the atomic and nuclear scale, thus forcing the use of QFT instead of QM.

\section{Conclusions}

We have looked for new ways of increasing the computation capacity by use of the characteristics of QFT representation that do not occur in the QM representation, but we were not able to find any such ways. However, we did determine that if Moore's law is to continue to be achievable in the future, then QFT will need to be used instead of QM.

Author Contributions: Conceptualization, N.L.; methodology, N.L.; writing-original draft preparation, N.L. and J.N.; writing - review and editing, N.L., J.N. and J.M.; supervision, J.N. and J.M.; project administration, J.M.; funding acquisition, J.M. All authors have read and agreed to the published version of the manuscript.

Funding: This work has been supported by "FCT-Fundação para a Ciência e Tecnologia" within the R\&D Units Project Scope: UIDB/00319/2020.

Institutional Review Board Statement: Not Applicable.

Informed Consent Statement: Not Applicable.

Data Availability Statement: Not Applicable.

Conflicts of Interest: The authors declare no conflict of interest. 


\section{Appendix A}

In QFT, the n-points correlation function for general state $|\Omega\rangle$ can always be represented using the general state two points correlation functions $\left\langle\Omega\left|T\left\{\psi_{\left(\vec{r}_{1}, t_{1}\right)} \bar{\psi}_{\left(\vec{r}_{2}, t_{2}\right)}\right\}\right| \Omega\right\rangle$ with $\bar{\psi}_{\left(\vec{r}_{2}, t_{2}\right)}=\psi_{\left(\vec{r}_{2}, t_{2}\right)}^{\dagger} \gamma^{0}$ and where + stands for conjugate transpose, and $\gamma^{0}$ is the $4 \times 4$ time-like Dirac matrix. A general state two-points correlation function can be expressed by perturbation series of the vacuum state $|0\rangle$ two-points correlation function combined with the interaction Lagrangian density $\mathcal{L}_{\text {int }}(\vec{r}, t)$. If $T$ is the time-ordering of the quantum fields $\psi(\vec{r}, t)$, then there is an equivalence [4] between the path integral (left side of Equation (A1)) and the canonical quantization (right side of Equation (A1)) approaches for calculating $\left\langle\Omega\left|T\left\{\psi_{\left(\overrightarrow{r_{1}}, t_{1}\right)} \bar{\psi}_{\left(\overrightarrow{r_{2}}, t_{2}\right)}\right\}\right| \Omega\right\rangle$ where $\varepsilon$ is an infinitesimal number:

$$
\lim _{\tau \rightarrow \infty(1-i \varepsilon)} \frac{\int D \psi \psi_{\left(\vec{r}_{1}, t_{1}\right)} \bar{\psi}_{\left(\overrightarrow{r_{2}}, t_{2}\right)} e^{i \int_{-\tau}^{\tau} d t \int_{-\tau c}^{\tau c} d^{3} \vec{r} \frac{\mathcal{L}_{\text {int }}(\vec{r}, t)}{\hbar}}}{\int D \psi e^{i \int_{-\tau}^{\tau} d t \int_{-\tau c}^{\tau c} d^{3} \vec{r} \frac{\mathcal{L}_{\text {int }}(\vec{r}, t)}{\hbar}}}=\lim _{\tau \rightarrow \infty(1-i \varepsilon)} \frac{\left\langle 0\left|T\left\{\psi_{\left(\vec{r}_{1}, t_{1}\right)} \bar{\psi}_{\left(\vec{r}_{2}, t_{2}\right)} e^{i \int_{-\tau}^{\tau} d t \int_{-\tau c}^{\tau c} d^{3} \vec{r} \frac{\mathcal{L}_{\text {int }}(\vec{r}, t)}{\hbar}}\right\}\right| 0\right\rangle}{\left\langle 0\left|T\left\{e^{i \int_{-\tau}^{\tau} d t \int_{-\tau c}^{\tau c} d^{3} \vec{r} \frac{\mathcal{L}_{\text {int }}(\vec{r}, t)}{\hbar}}\right\}\right| 0\right\rangle}
$$

The numerator of Equation (A1) is a product of the contributions by Feynman diagrams connecting the two spacetime locations $\left(\vec{r}_{1}, t_{1}\right)$ and $\left(\vec{r}_{2}, t_{2}\right)$ with the non-connecting diagrams, whereas the denominator is a contribution of only the non-connected diagrams; the division thus eliminates the contribution of the non-connected diagrams (which are proportional to the vacuum energy density) to Equation (A1) [4]. By dividing the spacetime into discrete spacetime chunks with $N$ elements for each spacetime orientation, it is obtained from Equation (A1) that for the canonical quantization approach:

$$
\approx \lim _{\tau \rightarrow \infty(1-i \varepsilon) N_{t}, N_{x}, N_{y}, N_{z} \rightarrow \infty} \prod_{j_{t}, j_{x}, j_{y}, j_{z}=1}^{N} \frac{\left\langle 0\left|T\left\{\psi_{\left(\overrightarrow{r_{1}}, t_{1}\right)} \bar{\psi}_{\left(\vec{r}_{2}, t_{2}\right)} e^{i \frac{\tau^{4} c^{3}}{N^{4}} \cdot \frac{\left.\left.\mathcal{L}_{(\vec{r}(j x, j y, j}\right), t\left(j_{t}\right)\right)}{\hbar}}\right\}\right| 0\right\rangle}{\left\langle 0\left|T\left\{e^{i \frac{\tau^{4} c^{3}}{N^{4}}} \cdot \frac{\mathcal{L}_{\left(\vec{r}(j x, j, j, j), t\left(j_{t}\right)\right)}}{\hbar}\right\}\right| 0\right\rangle}
$$

In QFT, the sum is done over all time-ordered configurations of the quantum fields which we call the "all configurations" sum; moreover, by assuming that spacetime coordinates $(\vec{r}, t)$ are flat, the phase associated to the contribution by the quantum fields at spacetime location $\left(\vec{r}^{\prime}, t^{\prime}\right)$ to the time-ordered transition between two quantum field configurations, $\psi_{(\vec{r}, t)}$ and $\bar{\psi}_{(\vec{r}, t+\Delta t)}$, is:

$$
\frac{\left\langle 0\left|\sum_{\text {all configurations }}\left\{\psi_{(\vec{r}, t+\Delta t)} \bar{\psi}_{(\vec{r}, t)} e^{i \frac{\Delta t}{\hbar}[c \cdot \Delta t]^{3} \mathcal{L}_{\text {int }}\left(\vec{r}^{\prime}, t^{\prime}\right)}\right\}\right| 0\right\rangle}{\left\langle 0\left|\sum_{\text {all configurations }}\left\{e^{i \frac{\Delta t}{\hbar}[c \cdot \Delta t]^{3} \mathcal{L}_{\text {int }}\left(\vec{r}^{\prime}, t^{\prime}\right)}\right\}\right| 0\right\rangle}
$$

\section{References}

1. Lori, N.F.; Neves, J.; Alves, V. Some considerations in quantum computing at subatomic scales and its impact in the future of Moore's law. Quantum Inf. Comput. 2020, 20, 1-13.

2. Ji, Z.; Natarajan, A.; Vidick, T.; Wright, J.; Yuen, H. MIP* = RE. arXiv 2020, arXiv:2001.04383v1. [CrossRef]

3. Yuen, H. The Shape of MIP* = RE. Quantum Frontiers. 2020. Available online: Quantumfrontiers.com/2020/03/01/the-shape-ofmip-re/ (accessed on 9 October 2020).

4. Peskin, M.E.; Schroeder, D.V. An Introduction to Quantum Field Theory (Frontiers in Physics), 1st ed.; Westview Press: Boulder, CO, USA, 1995.

5. Slofstra, W. The set of quantum correlations is not closed. arXiv 2017, arXiv:1703.08618. [CrossRef] 
6. Ozawa, N. Tsirelson's problem and asymptotically commuting unitary matrices. arXiv 2013, arXiv:1211.2712. [CrossRef]

7. Brune, M. Course 3 Cavity Quantum Electrodynamics; Estève, D., Raimond, J.-M., Dalibard, J., Eds.; Elsevier: Les Houches, France, 2004; Volume 79, pp. 161-185.

8. Alexoudi, T.; Kanellos, G.T.; Pleros, N. Optical RAM and integrated optical memories: A survey. Light Sci. Appl. 2020, 9, 91. [CrossRef] 